\title{
Metabolic Uncoupling: Biomass Control Strategy in Microbial Processes
}

\section{Gaurav Saini*}

Department of Civil Engineering, Sharda University, India

Keywords: Metabolic uncoupling; Chemical uncoupler; Biomass control; Activated sludge

\section{Editorial}

Microbial processes have been extensively used in engineered systems, esp. wastewater treatment, in situ bioremediation and most recently for biofuel production. While excess biomass may be desirable in some processes, it is detrimental to other systems including Activated Sludge Process (ASP), biofilters and subsurface bioremediation schemes, where uncontrolled microbial growth leads to increased cost, diminished efficiency and in extreme cases, process failure [1]. It has been reported by several researchers that a certain class of chemicals termed protonophores may be strategically employed to uncouple microbial metabolism. These lipophilic weak acids partially uncouple the catabolism from anabolism which leads to reduced growth yield, while maintaining the substrate utilization rate. This effectively leads to a control on biomass growth without decreasing the substrate consumption. This strategy has been beneficially employed in wastewater treatment to reduce the sludge quantity, without adversely affecting the treatment efficiency and sludge characteristics. Several chemicals have been studied to purposefully induce the uncoupling conditions in ASPs, including antibiotics and aromatic compounds. The more commonly tested uncouplers include 3, 3', 4', 5-tetrachlorosalicylanilide (TCS); 2, 4-dinitrophenol (DNP); pentachlorophenol (PCP); carbonyl cyanide m-chlorophenylhydrazone (CCCP); carbonyl cyanide p-(trifluoromethoxy) phenylhydrazone (FCCP); 2, 4, 6-trichlorophenol (TCP); rotenone; antimycin, etc. [2].

It is widely accepted that these chemicals have the ability to shuttle protons across the cell membrane and hence are named protonophores. This proton shuttling action effectively leads to a dissipation of Proton Motive Force (PMF). Under normal growth conditions, the catabolism and anabolism are coupled, i.e. the growth substrate breaks down and generates energy rich Adenosine Triphosphate (ATP) molecules through oxidative phosphorylation, a portion of which are used for cell maintenance and another portion is used (along with metabolites) to form cell biomass. However, under uncoupled conditions (brought on by chemical uncouplers), the catabolism is undisturbed leading to unchanged substrate consumption, while the ATP synthesis and anabolism slow down causing reduction in biomass yield. More specifically, these protonophores, in anionic form, trap the protons in low-pH environment outside the cell membrane and become neutral compounds, which then traverses across the membrane to the cell interior and release the proton in the high-pH environment. Afterwards the anionic form of the protonophore again crosses the cell membrane to return to its original position outside the cell and carries on this protonophoric activity [3]. The continuation of this uncoupling cycle leads to reduced proton-gradient across the membrane which results in low PMF, reduced ATP production and hence metabolic uncoupling.

Originally studied to elucidate the energy production in mitochondria, these chemicals have been extensively studied for reduction of biomass in wastewater treatment systems, esp. the activated sludge process. The ASP is employed in over $90 \%$ of the municipal wastewater treatment systems world-over. This process generates "waste" or "excess" sludge, biomass that needs to be disposed off and which requires more than $50 \%$ of the plant's operating cost [4]. Chemical uncouplers have been studied for possible biomass reduction in ASPs, while maintaining the organic removal rates and undisturbed sludge settleability. Up to $87 \%$ growth yield reduction has been observed in these studies. In a study involving four uncouplers, it was observed that $\mathrm{m}$-chlorophenol reduced the growth yield by $87 \%$ at a concentration of $0.15 \mathrm{mM}$ [5]. In another study, $78 \%$ growth yield reduction was observed at a TCS dosage of $2.3 \mu \mathrm{M}$ with no significant effect on substrate removal efficiency [6]. In another study of TCS application to ASP, excess biomass yield was reduced by $80 \%$ per day at a dose of $1 \%$ [7]. TCP reduced the sludge generation by $67 \%$, at a concentration of $1.2 \mathrm{mM}$, without affecting sludge settleability [8]. $0.03 \mathrm{mM}$ DNP demonstrated $21 \%$ reduction in biomass yield with 93\% COD removal for an ASP [9]. Full scale application of uncouplers has been reported at a wastewater treatment plant located in Phoenix, Arizona. Halogenated phenols were observed to cause reduced biomass yield, modest reduction in substrate uptake and increased oxygen consumption [10].

Chemical uncouplers have also been studied in other microbial systems, though not as frequently as compared to ASP. Fixed film reactors, such as trickling filters or membrane bioreactors, require a control on biomass accumulation to prevent bioclogging of reactors. $\mathrm{DNP}$, at a dose of $0.05 \mathrm{mM}$, exhibited $65 \%$ biofouling reduction in a membrane bioreactor employed in wastewater treatment [11]. In a recent study on fixed biofilm reactors, up to $85 \%$ increase in surface elimination capacity for toluene degrader Stenotrophomonas maltophilia was observed when using PCP as an uncoupler [2].

Similarly, very few chemical uncoupling studies have been carried for other microbial processes of interest. In situ bioremediation schemes can suffer significant adversity by bioclogging of soil pores, which leads to reduction in hydraulic conductivity thereby decreasing the substrate transport from injection wells to the locations of interest [12]. In extreme cases, such lack of control over biomass growth can cause shift in dominant microbial community and complete failure of remediation scheme [13]. In a study on Shewanella oneidensis MR-1, which is a model microbe for subsurface metal bioremediation studies, TCS application resulted in 53\% biomass yield reduction for $2 \mathrm{mM}$ uncoupler dose [1]. Another microbial application of interest is the Microbial Fuel Cell (MFC), device which can generate either hydrogen

*Corresponding author: Gaurav Saini, Assistant Professor, Department of Civil Engineering, Sharda University, Plot No. 32-34, Knowledge Park-3, Greater Noida, Uttar Pradesh 201306, India, Tel: (91) 828-7051260; E-mail: gaurav. saini@sharda.ac.in

Received August 04, 2014; Accepted August 05, 2014; Published August 12 2014

Citation: Saini G (2014) Metabolic Uncoupling: Biomass Control Strategy in Microbial Processes. J Microb Biochem Technol 6: e117. doi:10.4172/1948 5948.1000e117

Copyright: (c) 2014 Saini G. This is an open-access article distributed under the terms of the Creative Commons Attribution License, which permits unrestricted use, distribution, and reproduction in any medium, provided the original author and source are credited 
or electricity while treating wastewater or other organic waste. Limited number of studies have focused on the application of chemical uncouplers or inhibitors in MFCs. Current generation in these devices is generally constrained by high concentrations of organic substrate and electron acceptors [14]. Such devices may fruitfully employ uncouplers or inhibitors to control the biomass growth while dealing with the excess substrates. Working on a hypothesis that uncouplers will increase the current yield during aerobic respiration, researchers found that $0.24 \mathrm{mM}$ of DNP completely inhibited the electrochemical activity [15]. More research is needed in these areas, in situ bioremediation and MFCs, to ensure beneficial applications of chemical uncouplers.

The effectiveness of chemical uncouplers in reducing excess sludge production has been proven by numerous studies and actual fieldscale application. Similar results have also been reported for other microbial processes of interest. However, there are still few issues with the application of these chemicals to microbial systems. Chief among these are the xenobiotic nature of these compounds, uncertainty over potential adaptation of microbes during long-term application and potentially harmful effects of these chemicals on human exposure. Aromatic uncouplers, esp. DNP, TCP and PCP are EPA priority pollutants, with TCP and PCP listed as probable carcinogens [16]. There are regulatory limits on concentration of these chemicals in surface waters. Potential environmental release and/or human exposure of these chemicals from wastewater treatment plant during effluent disposal are thus challenges that need to be considered.

Not all the chemical uncouplers are harmful to the environment. Some of these have been used in low concentrations in products that are already used by us. TCS is a component of soaps, shampoos, rinses and is also used as preservative in textile finishes and certain petroleum products. An effective strategy, thus, would be to either use environmentally friendlier uncouplers like TCS or use other uncouplers at extremely low concentrations and prevent their accumulation in the microbial systems.

There is no doubt over the efficacy of chemical uncouplers in controlling biomass yield in growth systems, esp. wastewater treatment, and their potential beneficial applications to other microbial systems. However, more research is needed, esp. with pure cultures and in fixed growth systems, before these chemicals can be strategically employed to control the biomass during various microbial processes.

\section{References}

1. Saini G, Wood BD (2008) Metabolic uncoupling of Shewanella oneidensis MR-1, under the influence of excess substrate and 3, 3', 4', 5-tetrachlorosalicylanilide (TCS). Biotechnol Bioeng 99: 1352-1360.

2. Detchanamurthy S (2013) Impact of Different metabolic uncouplers on the specific degradation rate of toluene in a differential biofiltration reactor. PhD Thesis, University of Canterbury.

3. Detchanamurthy S, Gostomski PA(2012) Metabolic uncouplers in environmental research-A critical review. Rev Chem Eng 28: 307-317.

4. Liu Y (2003) Chemically reduced excess sludge production in the activated sludge process. Chemosphere 50: 1-7.

5. Yang XF, Xie ML, Liu Y (2003) Metabolic uncouplers reduce excess sludge production in an activated sludge process. Process Biochem 38:1373-1377.

6. MacLeod R, Wisse G, Stejskal F (1988) Sensitivity of some marine bacteria, a moderate halophile, and Escherichia coli to uncouplers at alkaline $\mathrm{pH} . \mathrm{J}$ Bacteriol 170: 4330-4337.

7. Hassani A, Nejaei A, Torabian A (2011) Excess sludge minimization in conventional activated sludge pilot plant by three chemical matters. Int J Environ Res 5: 981-988.

8. Tao W, Chengquan $Y$, Weimin $L$, Haiwen $X$, Jing $L(2010)$ Effect of 2, 4, 5 trichlorophenol on A 2/O process sludge yields. Environ Sci Mgmt 35: 38-41.

9. Chen GW, Yu HQ, Xi PG, Xu DQ (2008) Modeling the yield of activated sludge in the presence of 2, 4-dinitrophenol. Biochem Eng J 40: 150-156.

10. Okey RW, Stensel HD (1993) Uncouplers and activated sludge-the impact on synthesis and respiration. Toxicol Environ Chem 40: 235-254

11. Xu H, Liu Y (2011) Control and cleaning of membrane biofouling by energy uncoupling and cellular communication. Environ Sci Technol 45: 595-601.

12. Seki K, Thullner M, Hanada J, Miyazaki T (2006) Moderate bioclogging leading to preferrential flow paths in biobarriers. Ground Water Monit Remediation 26 : 68-76.

13. Anderson RT, Vrionis HA, Ortiz-Bernad I, Resch CT, Long PE, et al. (2003) Stimulating the in situ activity of Geobacter species to remove uranium from the groundwater of a uranium-contaminated aquifer. Appl Environ Microbiol 69(10): 5884-5891.

14. Rabaey K, Verstraete W (2005) Microbial fuel cells: novel biotechnology for energy generation. Trends Biotechnol 23: 291-298.

15. Kim BH, Park HS, Kim HJ, Kim GT, Chang IS, et al. (2004) Enrichment of microbial community generating electricity using a fuel-cell-type electrochemical cell. Appl Microbiol Biotechnol 63: 672-681.

16. Paul E, Liu Y (2012) Biological Sludge Minimization and Biomaterials/Bioenergy Recovery Technologies. John Wiley and Sons. 